\title{
Implementación de los PLE, ¿necesidad perentoria en la semipresencialidad, para promover la construcción colectiva del conocimiento?
}

\section{Implementation of PLE, ¿urgent need in blended learning, to promote the collective construction of knowledge?}

\author{
Elba Rosa Gómez Barajas ${ }^{1}$, Luis Armando Negrete Salazar ${ }^{2}$ \\ ${ }^{1}$ Departamento de Ciencias Económico-Admistrativas, Universidad de Guadalajara, México (elbagomez@ valles.udg.mx) \\ ${ }^{2}$ Departamento de Ciencias Computacionales e Ingenierías, Universidad de Guadalajara, México \\ (luis.negrete@valles.udg.mx)
}

Recibido el 10 de noviembre de 2015; revisado el 18 de noviembre de 2015; aceptado el 18 de noviembre de 2015; publicado el 14 de diciembre de 2015

\section{RESUMEN:}

El presente artículo es una descripción teórica en el contexto del Centro Universitario de los Valles de la Universidad de Guadalajara. En esta investigación que aún no tienen resultados contundentes, se describen algunas de las problemáticas actuales que del uso de la Plataforma Moodle se detectaron en el alumnado en la Modalidad Educativa Semipresencial del CUValles. Se encuentra bastante interesante sumergirse en el mundo de los PLE (Entornos Personales de Aprendizaje), como una manera de promover la heterogeneidad de herramientas disponibles en el Internet, para que el alumno elija entre la gran diversidad de dispositivos, contenidos, almacenajes, modos de interacción y trabajo colectivo, de acuerdo a su manera propia de aprender. Se describen algunas de las competencias que mediante la utilización de los PLE, pueden adquirir los estudiantes, basados en los preceptos de las tecnologías emergentes. En un estudio diagnóstico previo, realizado en 2014 por tres investigadores del Laboratorio de Innovación y Calidad Educativa, se encontró que los alumnos no utilizan muchas de las herramientas propias de la Moodle. Esta es utilizada para el envío de tareas (repositorio), más que para actividades de coconstrucción del conocimiento e intercambio de información. No es utilizada para la interacción online y offline entre profesores y alumnos, alumnos-alumnos, etc. Es, pues, una forma de trabajar de manera más individualizada y poco lúdica, y con una casi nula posibilidad de promover el aprendizaje significativo, la autogestión y los proyectos por medio de la inteligencia colectiva. En el Centro Universitario de los Valles se utiliza la plataforma Moodle de manera institucionalizada, es decir que es la oficialmente reconocida para que alumnos y profesores interaccionen y complementen las actividades que se realizan en el cara a cara, y por tanto el resto de los días convergen en este LMS (Learning Management System)
PALABRAS
CLAVE:
ENTORNOS
PERSONALES
DE
APRENDIZAJE,
EDUCACIÓN SUPERIOR, TIC, EDUCACIÓN SEMIPRESENCIAL.

\begin{abstract}
:
This article is a theoretical description in the context of the university center of valleys of the University of Guadalajara. In this research are not yet conclusive results are described some of the current problems that the use of the moodle platform were detected in students in a blended mode of CUValles education. It is quite interesting to dive into the world of PLE (personal learning environments), as a way to promote the diversity of tools available on the internet, so that students choose the wide variety
\end{abstract}


of devices, content, storage, interaction modes and collective work, according to their own way of learning. Some of the powers that using the PLE, students can acquire based on the precepts of emerging technologies are described. In a previous diagnostic study, conducted in 2014 by three researchers from the laboratory of educational innovation and quality it found that students do not use many of the tools themselves moodle. It's used for sending tasks (repository), rather than for activities co-construction of knowledge and exchange of information. It's not used for on-line and off-line interaction between teachers and students, student-student, etc. it is therefore a way of working much more individualized and little playful way, and almost no possibility of promoting meaningful learning, self-management and projects through collective intelligence. In the university center of valleys moodle institutionalized way it is used, it's the officially recognized for students and interact teachers and complement the activities carried out in the face to face, and therefore the rest of the day converge in this LMS (learning management system).

\section{KEYWORDS: PERSONAL LEARNING ENVIRONMENTS, HIGHER EDUCATION, TIC, BLENDED LEARNING.}

\section{INTRODUCCIÓN}

La educación a nivel mundial es uno de los bienes más preciados, el único capaz de erradicar la pobreza y de formar capital humano pensante, crítico, propositivo e innovador.

En este actual escenario donde las Tecnologías de la Información y de la Comunicación (TIC) están impactando en todos los sectores de la sociedad, es paradójico que a pesar de que en la mayorías de las instituciones de educación superior (IES), donde existe una interconectividad social, no haya una producción colectiva del conocimiento de quienes se encuentran en las aulas de una IES, que no se promuevan las estrategias pertinentes para favorecer que el conocimiento sea el producto de la inteligencia colectiva.

Para Garrison y Anderson (2005, citado en Estrada, Catañeda y Padilla, 2012, p. 2), "sobre las comunidades de indagación hace énfasis en cómo las tecnologías y en particular las comunidades virtuales de aprendizaje, apoyan la construcción conjunta del conocimiento".

La educación superior tiene como objetivo hacer eco a las tendencias actuales en materia económica, social, cultural, política, medioambiental, tecnológica, etc., y por consecuencia estructurar propuestas para la resolución de problemas del presente, y seguir generando una sustentabilidad holística, de forma tal que se cree un mejor escenario para que las generaciones futuras, tengan las bases para continuar con el crecimiento de los diferentes sectores de una sociedad, en equidad, para ello, cuenta con el valioso y transformador fenómeno de la generación de conocimiento (UNESCO, 2005; UdG, 2014).

La Universidad de Guadalajara (UdG), como universidad pública, dentro de su Plan de Desarrollo Institucional (2014, p. 13), dice: "El valor económico del conocimiento y la posesión de ciertas habilidades y destrezas demandadas en los mercados laborales han impulsado un vertiginoso aumento en la matrícula de educación superior".

Además, nuestra Universidad a través de sus ejes estratégicos de docencia, investigación y extensión, es capaz de atender demandas locales, regionales, nacionales e internacionales.

Dicho lo anterior, la misma UdG, en su Plan de Desarrollo Institucional (PDI 2014-2030), enfoca sus estrategias en la contribución del desarrollo y oferta de programas educativos no convencionales, basados en el uso de las TIC.

Por tanto, el aumento de la matrícula puede ser solventado con modalidades de enseñanza no convencional, como la semiprencial, para dar respuesta al aumento de los alumnos que ingresan hoy en día al nivel de educación superior (Horizon, 2007; 2010; 2015).

Los centros regionales de la UdG se encuentran inmersos en un contexto descentralizado de la zona metropolitana de Guadalajara, y tomando en cuenta las características de cada una de las poblaciones sobre las que puede influir, educando y formando sujetos, es que determina el tipo de acciones y estrategias para la resolución de problemas de toda índole y la propuesta de mecanismos de cambio e innovación.

El conocimiento no es estático, está en constante cambio y movimiento; es por ello que crear nuevo conocimiento no es ya una quimera, es meramente la esencia de transformar la información (UNESCO, 2008).

En la actualidad, la vorágine de la tecnología se está viendo reflejada tanto en el número de personas que tienen acceso a ella, como en la gran diversidad de herramientas de distinta índole a disposición del usuario.

En el caso del estudiante de las IES, está envuelto en un escenario digital que le permite hacer 
sus propias e individualizadas elecciones de los dispositivos, los espacios web, las redes sociales, y muchos de los mass-media (o medios de masas) que el mundo actual pone a su disposición, siempre y cuando se adapte a sus propias maneras de aprender, a sus intereses propios y a las habilidades que tiene o va desarrollando, a la par de su interacción con el inmenso mundo de la Web 2.0.

Si queremos referirnos a la educación 2.0, viene ad-hoc la definición de De Haro (2007), la cual es retomada por Navas (2013, pp. 97-98), donde afirma:

...nos define la educación 2.0 como aquella que permite al alumno a prepararse para incorporarse a una sociedad, donde la cultura del aprendizaje deber estar presente a lo largo de toda su vida profesional. Y agrega, muchos de los conocimientos que el estudiante adquiere en sus años de escolaridad formal, dejarán de ser útiles unos pocos años después de haber egresado de la universidad. Es por ello que se hace necesario que esté preparado para gestionar por sí mismo, una formación continua a lo largo de su vida como profesional e incluso más allá.

Es justo porque en esta revolución digital actual se crea un panorama social, político, cultural distinto, es por ello que se demanda la existencia de un nuevo perfil de profesionales de la educación, individuos y grupos que trasciendan a diversos ámbitos por medio de la interacción tecnológicas en toda su caleidoscópica extensión.

\subsection{Material y método}

Estudio previo a la actual investigación: Estudio de la opinión de los estudiantes, del Centro Universitario de los Valles

En el año 2014 se llevó a cabo una investigación denominada "Estudio de la opinión de los estudiantes, del Centro Universitario de los Valles sobre aspectos de la docencia y servicios académicos, en el marco de la Evaluación Institucional del CUValles", con el objeto de conocer la perspectiva del alumno en relación a la modalidad semipresencial en varios aspectos, uno de los cuales fue el uso de la Plataforma Moodle, de la que hablaremos en este artículo, destacando únicamente algunas herramientas de la misma. Moodle es la plataforma que se utiliza de forma institucionalmente oficializada, para complementar las sesiones presenciales ( 2 o 3 días) y para la realización de actividades cuando no están en el cara a cara con el profesorado (5 días). Como es evidente, es mayor la necesidad de la actividad virtual que la presencial.

La metodología utilizada fue tipo encuesta, con metodología Limesurvey.

De acuerdo a HORIZON 2015, una de las tendencias educativas, es el aumento de la enseñanza híbrida. El uso de las tecnologías emergentes (TE), debe adecuarse a las necesidades específicas de los estudiantes y no a las del profesor o las políticas de la IES, el estudiante es el usuario y el ente más importante de todo el proceso educativo (Boudé, 2013; Horizon, 2015).

Por otra parte, hacemos especial hincapié en que el docente del aprendizaje híbrido o blended learning, debe encontrar la manera de fomentar en el alumnado la interacción social y propiciar el pensamiento crítico a través de actividades en línea, de manera equitativa a como se logra en el cara a cara.

Hibridar el aprendizaje o la enseñanza no es tarea fácil, y justo por ello es que en las modalidades semipresenciales, como la del Centro Universitario de los Valles, es menester la realización de estudios diagnósticos sobre la utilización de las TIC, partiendo de la idea de que el alumnado debe contar con el apoyo del profesor, y de la institución en general para darle libertad de elegir de manera personal, cómo y con qué herramientas quiere aprender.

\section{RESULTADOS}

Basándonos en el estudio previo realizado en el CUValles en el año 2014, realizado por Ríos, Gómez y Pasillas, tenemos los siguientes datos, respecto al uso que se hace de la plataforma Moodle.

El cuestionario fue contestado por 1755 estudiantes, que supone el $51 \%$ de los estudiantes, de las once licenciaturas y que estaban matriculados en el curso 2013 B. Los mayores porcentajes corresponden a los estudiantes de Psicología con el 63.1\% ; Administración con $58.1 \%$ y los menores porcentajes lo tienen los estudiantes de Abogado con el $45.8 \%$ y los de Educación con el $44.4 \%$.

La muestra de estudiantes de CUValles se ha distribuido entre un $56.9 \%$ de mujeres y un $43.1 \%$ de hombres. No obstante, la distribución de género presenta importantes diferencias en función de las licenciaturas. Así, tenemos algunas con una fuerte presencia de mujeres como son Trabajo Social con el $92.5 \%$, Psicología con el $81.7 \%$ y Educación con el 78.6\%; y otras con una gran concentración de hombres como son Mecatrónica con el 93.4\%, 
Electrónica con el $78.3 \%$ y Agronegocios con el $78 \%$.

El $85.1 \%$, de los estudiantes de Educación, afirma que usan la plataforma para consultar lecturas, el porcentaje de la muestra total de alumnos de CUValles es el $74 \%$, un $11.1 \%$ menos. El mayor porcentaje de este uso corresponde a los estudiantes de Psicología con el $86.5 \%$, y el menor porcentaje a los estudiantes de Abogado con un $62.3 \%$, el $24.2 \%$ menos. Estos datos reflejan una diferencia importante en el uso de Moodle, para consultar lecturas, respecto a los estudiantes de las licenciaturas. La licenciatura de Educación tiene el segundo mayor porcentaje.

El 98.2\% de los estudiantes de CUValles usan la plataforma para subir tareas. El 100\% de los estudiantes de Administración, Electrónica y Mecatrónica afirman que suben tareas en la plataforma Moodle. Los estudiantes que menos usan la plataforma para subir tareas son los de Turismo con el 94.7\%, Sistemas con el $96.3 \%$ y Agronegocios con el 97\%. Las diferencias de uso, entre las licenciaturas, es pequeña y están en un intervalo del $5.3 \%$, entre el mayor y el menor porcentaje.

Los foros son utilizados por el $67.7 \%$ de la muestra de alumnos de CUValles. Los mayores porcentajes de uso corresponden a los estudiantes de Educación con un 81.2\%, Administración con el $75.2 \%$ y Contaduría con el $74.1 \%$; y los menores son los de Agronegocios con el 44.7\%, Trabajo Social con el 54.2\% y Electrónica con el 54.7\%. El intervalo entre el mayor y el menor porcentaje es del $36.5 \%$, siendo un intervalo muy amplio que evidencia una gran diferencia en el uso de los foros.

Los chats son unas herramientas muy poco utilizadas por los estudiantes, solo el $9.9 \%$ de los estudiantes, de la muestra de alumnos de CUValles, afirma que los usa en la plataforma Moodle. Las licenciaturas que más usan los chats son Electrónica con el 14.2\%, Administración con el $12.8 \%$ y Abogado con el $12.1 \%$; y las que menos lo usan son Sistemas con el $6.5 \%$, Mecatrónica con el $6.6 \%$ y Agronegocios con el $7.6 \%$. Las diferencias porcentuales del uso de los chats varían entre el mayor porcentaje que corresponde a los estudiantes de Electrónica y el menor de Sistemas de Información en un $7.7 \%$.

Las wiki casi no son usadas, la media de utilización del total de la muestra del alumnado de CUValles es un $2.8 \%$, lo que indica un uso marginal. La wiki es la herramienta, incluida en la plataforma Moodle, menos utilizada por los estudiantes de CUValles.
El uso de las sopas de letras es poco relevante. El porcentaje, de la muestra total de alumnos de CUValles, que afirma utilizarla es el $4.7 \%$.

El porcentaje, de la muestra de alumnos de CUValles, que afirma que ha utilizado "hot potatoes" es el 3.8\%. Las licenciaturas con mayores porcentajes de uso son la de Electrónica con el 9.4\%, Contaduría con el $6.9 \%$ y Educación con el $6.5 \%$; y las que tienen los menores porcentajes son Trabajo Social con el $0.8 \%$, y Turismo y Agronegocios, ambas con el 1.5\%.

El 24.3\% de los estudiantes, de la muestra de CUValles, creen que las actividades en línea sustituyen la presencialidad. Este porcentaje evidencia una falta de confianza en las actividades en línea, de cara a compensar la presencialidad. Es muy importante resaltar que más del $75 \%$ de los estudiantes no creen que las actividades en línea compensan la presencialidad, lo que nos lleva a inferir que asumen por motivos personales el modelo de CUValles, siendo conscientes de que tienen un perjuicio por la falta de funcionamiento de las actividades en línea.

\section{DISCUSIÓN}

Los resultados anteriormente descritos, nos arrojan una muy precaria utilización de la moodle para la autogestión y el aprendizaje colaborativo, es muy evidente la casi nulidad de las wikis, hot potatoes, sopa de letras, incluso el foro mismo, y por tanto enfatizamos que no se están promoviendo en el alumno, las dos competencias anteriormente mencionadas.

Estimamos que las TE pueden ser una buena opción, aunque puede haber dificultades.

\begin{abstract}
Las tecnologías emergentes son herramientas, conceptos, innovaciones y avances utilizados en diversos contextos educativos al servicio de diversos propósitos relacionados con la educación. Además, propongo que las tecnologías emergentes ("nuevas" y "viejas") son organismos en evolución que experimentan ciclos de sobre expectación y, al tiempo que son potencialmente disruptivas, todavía no han sido completamente comprendidas ni tampoco suficientemente investigadas (Adell y Catañeda, 2010, citado en Valestsianos 2012, p. 16).
\end{abstract}

No obstante la definición anterior, cuando se habla de TE se hace alusión a tecnologías que no son del todo difundidas o aplicadas por un gran número de personas; no significa, por tanto, que no existan, pues son difundidas por institutos, informes 
como INTEF (Instituto Nacional de Tecnologías Educativas y de Formación del Profesorado) y, sobre todo, los regulares Informes Horizon donde se destacan las tendencias del uso pedagógico de la tecnología, cómo se usan, dónde se usan, cuáles deberían utilizarse e incluso las que se utilizarán en el futuro. A esta iniciativa de publicar e informar sobre la futurista utilización de las TIC se suman los informes JISC o la documentación reciente del FUTURELAB.

Dentro de las TE de acuerdo a Horizon 2007, tenemos las siguientes:

1. Computación en la nube

2. Inteligencia colectiva

3. Mashups de datos

4. Webs en colaboración

5. Redes sociales

Todas tienen que ver con las posibilidades que la tecnología brinda a través de dispositivos, de software y hardware que posibilitan tanto al estudiante y al profesor el almacenaje, procesamiento, disposición de recursos, remezcla de información y herramientas diversas albergados en la web, diseño de estrategias didácticas y su consecuente implementación.

¿Qué tipo de habilidades se propician con este tipo de TE? por mencionar algunas (basadas en la información de Horizon desde 2007 hasta el 2015):

a) Construcción colectiva del conocimiento.

b) Innovación educativa y transdisciplinary.

c) Desarrollo de CMI (competencias en manejo de información).

d) Inter y transdiscpilinariedad, co-construcción del conocimiento.

e) Potencializar las habilidades sociocomunicativas a través de la red (redes sociales, mashups de datos).

f) Proyectos conjuntos donde se desarrollen las competencias básicas de escritura, pero a un nivel de colectividad.

g) La interacción de las reflexiones, aprendizaje significativo, la autogestión y la capacidad crítica de cada individuo, eslabonándose hacia una inteligencia colectiva como un sistema complejo para la realización de proyectos.

Dentro de las anteriormente mencionadas TE, focalizaremos ahora a los PLE, ya que entran en las cinco TE.

Cabero (2013, p. 15), a través del estudio presentado por el Centre for educational technology \& interoperability standars (CETIS. 2007), asevera que:

El PLE no está directamente relacionado con la enseñanza y el aprendizaje, su función principal es que el alumno se coordine con la tecnología. Proporciona la apertura a nuevos canales de coordinación entre la vida y el aprendizaje. La intervención del PLE sitúa a la tecnología con el aprendizaje, no con la institución. Es el alumno quien debe ser responsable de la coordinación de las diferentes tecnologías que tiene a su disposición para hacer frente a su aprendizaje.

Y que adicionalmente (opus cit) "la vertiginosa aparición de nuevos sistemas informáticos, actualizaciones, etc. hacen que el usuario debe estar en constante proceso de adaptación".

Barroso, Cabero y Vázquez (2012, p. 4), aseveran que "el conocimiento en un LMS es estático, declarativo (aseverativo), y basado en la autoridad. Elementos, que ciertamente, distan mucho de promover el aprendizaje creativo y significativo".

Creemos que la flexibilidad que ofrecen los PLE van justamente acordes a lo que el Modelo CUValles pretende, "flexibilizar la educación de sus estudiantes" mediante el modelo educativo. Afirmamos que se debe dar paso a la innovación tecnológica, hacer uso de las herramientas Web 2.0, las cuales podrían favorecer y ampliar las posibilidades de capacidad autogestión del alumnado, impulsar la interacción en grupo, adaptarse a sus formas particulares de aprender ofreciéndole la posibilidad de utilizar entornos de aprendizaje, que se centren en las necesidades personales.

Además, con el uso de los PLE, mediante un estudio experimental, se pueden tener datos para hacer nuevas propuestas de utilización de las TIC, de una manera mucho más amplia, dentro del CUValles. La investigación validaría el efecto que causa en los alumnos estas herramientas si se implementan, además de apreciar las competencias que se alcanzarían como resultado del uso cotidiano dentro de alguna asignatura y su práctica en otros entornos de aprendizaje distintos a la Moodle.

Es justo aquí donde los PLE se convierten en la manera más eficaz de que el usuario se coordine con la tecnología y transite por distintos senderos no solo del aprendizaje sino de su vida personal (Cabero y Vázquez-Martínez; 2013).

Dentro del mundo de las TIC, en las que prima la socialización, encontramos a los PLE como unos de los más innovadores espacios elegidos por el alumno para realizar acciones pedagógico- 
personales tales como: accesar a la información, procesarla mediante la reflexión, compartirla mediante la interacción con profesores, alumnos y los propios contenidos.

Campos (2008, citado por Cabero y Vázquez, 2013 , p. 13), indica que las características que define la red social son:

Concepto de comunidad, a través de la creación de redes de usuarios que interactúan, dialogan y aportan comunicación y conocimiento; tecnología flexible y ancho de banda necesario para el intercambio de información y estándares web de aplicación libre; y una arquitectura modular que favorece la creación de aplicaciones complejas de forma más rápida, a un menor coste. (...) en la web 2.0 producen contenidos (blogosfera), participan del valor (intercambio) y colaboran en el desarrollo de la tecnología. El proceso de comunicación genera, en definitiva, un flujo activo de participación.

\section{CONCLUSIONES}

La educación semipresencial hibrida dos tipos de enseñanza-aprendizaje: la presencial y la virtual; por ello es urgente y muy prioritario que el profesorado del Centro Universitario de los Valles de la Universidad de Guadalajara cuente con una constante formación en el uso de las TE.

No solo es suficiente con el manejo y dominio de la tecnología. El profesorado debe tener una actitud de apertura a la innovación pedagógica, es decir, que no solo por utilizar TE se garantiza la libertad que tiene que darle al alumno de elegir su propio PLE. La heterogeneidad de perfiles del estudiante exige del profesor las competencias de ser innovador en sus procederes, actitudes flexibles, habilidades socio-afectivas y comunicativas y respeto a las opiniones diversas.

El uso de la plataforma Moodle dentro del Centro Universitarios de los Valles, a decir por el alumnado, no sustituye a las sesiones presenciales. Más del $75 \%$ de los encuestados no se sienten satisfechos con la actividades en línea que se realizan en las sesiones virtuales.

Las principales herramientas que se utilizan de la Moodle son para "consulta de lecturas" y para "subir tareas", lo que la convierte en un instrumento estático y casi unidireccional, pues en otra parte del mismo estudio la mayoría de los encuestados afirmaron que no reciben retroalimentación de las tareas realizadas.

En cuanto a la interacción social, el intercambio de reflexiones individuales que podrían trascender al aprendizaje colectivo, solamente se utilizan los foros, es decir comunicación offline con un máximo del $67 \%$ de la muestra total.

La interacción online por medio de los chats es prácticamente nula, ya que solo un $9.9 \%$ del total de alumnos encuestados afirmaron utilizarla en sus asignaturas.

Otro tipo de herramientas como las wiki, Hot Potatoes y la sopa de letras son infrautilizadas, con un $2.8 \%, 3.8 \%$ y $4.7 \%$ respectivamente.

Un modelo educativo centrado en el aprendizaje, como dice el Dictamen de Creación del Centro Universitario de los Valles, desde hace 15 años, y donde el alumno asiste una sola vez a la semana, dos horas presenciales, a cada asignatura, necesita hacer una parada forzosa para la reflexión.

El uso de los PLE podría darnos resultados esclarecedores de lo que en realidad los alumnos aprenden en la actual LMS (Moodle), las herramientas utilizadas y elegidas de acuerdo a sus ritmos de aprendizaje, modos de aprender, de interactuar con el contenido, con el compañero y con el profesor, para ello se aplicaría un post-test para medir las competencias adquiridas al utilizar los PLE.

La innovación educativa, las TE, la megadiversidad de dispositivos, estrategias, plataformas, apps, etc., no pueden ser ignorados por la educación superior, donde tiene cabida y debiera tener una perentoria presencia el desarrollo de las competencias personales y comunicativas, las CMI (Competencias en el Manejo de la Información), la capacidad crítica y reflexiva, la co-contrucción del conocimiento y el impulso hacia el aprendizaje complejo y colectivo.

Las TIC en esta nueva etapa digital y su innegable carácter sinérgico debe marcar un nuevo sendero, que tanto profesores, alumnos y la misma IES, transite para dejar atrás las sesgadas visiones de unificar las plataformas tecnológicas, dando paso así a la multifurcación de aplicaciones y herramientas, para enriquecer el proceso de convertir la información en conocimiento colectivo e inteligencia institucional.

\section{REFERENCIAS}

Adell, J., y Castañeda, L. (2012). Tecnologías emergentes, ¿pedagogías emergentes? En J. Hernández, M. Pannesi, D. Sobrino, y A. Vázquez (Coords.). Tendencias Emergentes en Educación con TIC (pp. 1332). Barcelona: Asociación Espiral, Educación y Tecnología. Recuperado de https://digitum.um.es/jspui/bitstream/10201/29916/1/Ad ell_Castaneda_emergentes2012.pdf 
Barroso, J.; Cabero, J. y Vázquez, A. I. (2012). La formación desde la perspectiva de los entornos personales de aprendizaje (PLE). Apertura. Revista de innovación educativa, 4(1), 1-12. Recuperado de http://www.udgvirtual.udg.mx/apertura/index.php/apert ura3/article/view/209/224

Boudé, O. (2013). Tecnologías emergentes en la educación: una experiencia de formación de docentes que fomenta el diseño de ambientes de aprendizaje. Educação \& Sociedade, 123(34), 531-548. Recuperado de http://www.redalyc.org/pdf/873/87328002014.pdf

Cabero, J.; Barroso, J., y Llorente, M. C. (2010). El diseño de Entornos Personales de Aprendizaje y la formación de profesores en TIC. Digital Education Review, 18, 26-37. Recuperado http://www.raco.cat/index.php/DER/article/view/21853 $1 / 297805$

Cabero, J., y Vázquez, A. I. (2013). Los Entornos Personales de Aprendizaje: uniendo lo formal, informal y no formal para la construcción del conocimiento. En M. C. Fonseca (Coord.), Los Entonos Personales de Aprendizaje. Visiones y retos para la formación (pp. 926). Recuperado de http://tecnologiaedu.us.es/tecnoedu/images/stories/ples_ 2014.pdf

Estrada, J. A., Hernández, R., y Padilla, S. (2012). La construcción colectiva del conocimiento en los foros de discusión. Un estudio de caso. Diálogos sobre Educación, 5, 1-18. Recuperado de http://www.revistadialogos.cucsh.udg.mx/sites/default/fi les/dse_a3_n5_jul-dic2012_estrada.pdf

New Media Consortium y Universitat Oberta de Catalunya (2007). Informe Horizon 2007. Recuperado de http://www.uoc.edu/portal/_resources/CA/documents/in novacio/horizon_report_2007_es.pdf

New Media Consortium y Universitat Oberta de Catalunya (2010). Informe Horizon 2010. Recuperado http://www.nmc.org/pdf/2010-Horizon-Report-es.pdf

NMC (2015). Horizon Report Edición Educación Superior 2015. Recuperado de http://cdn.nmc.org/media/2015nmc-horizon-report-HE-ES.pdf

Navas, E. (2013). Los entornos personales de aprendizaje en el marco de la educación permanente. Revista de Educación Mediática y TIC, 2(1), 94-110. Recuperado de http://www.edmetic.es/Documentos/Vol2Num12013/6.pdf

Ríos, J. M., Gómez, E. R., y Pasillas, D. G. (2014). Estudio de la opinión de los estudiantes, del Centro Universitario de los Valles sobre aspectos de la docencia y Servicios Académicos, en el marco de la Evaluación Institucional del CUValles. Inédito.

UNESCO (2005). Hacia las Sociedad del Conocimiento. Informe Mundial de la UNESCO. Recuperado de http://unesdoc.unesco.org/images/0014/001419/141908s .pdf

UNESCO (2008). Educación Superior y Sociedad. El movimiento de responsabilidad social de la universidad: una comprensión novedosa de la misión universitaria. Recuperado http://www.iesalc.unesco.org.ve/dmdocuments/bibliotec a/libros/ess_septiembreao_13_nro2_2008.pdf

Universidad de Guadalajara (2000). Principios pedagógicos del modelo Académico del CUValles. Recuperado de http://www.web.valles.udg.mx/vallesweb/sites/default/fi les/canales/01_conoce_cuvalles/modelo_academico_cu valles.pdf
Universidad de Guadalajara (2014). Plan de Desarrollo Institucional 2014-2030. Construyendo el futuro. Recuperado de http://www.copladi.udg.mx/content/pdi2014-2030 